Appl. Set-Valued Anal. Optim. 1 (2019), No. 2, pp. 171-185

Available online at http://asvao.biemdas.com

https://doi.org/10.23952/asvao.1.2019.2.06

\title{
AN ITERATIVE ALGORITHM FOR SOLVING EQUILIBRIUM PROBLEMS, VARIATIONAL INEQUALITIES AND FIXED POINT PROBLEMS OF MULTIVALUED QUASI-NONEXPANSIVE MAPPINGS
}

\author{
T.M.M. SOW \\ Department of Mathematics, Gaston Berger University, Saint Louis, Senegal
}

\begin{abstract}
The purpose of this paper is to introduce and study an iterative algorithm, which is based on an extragradient algorithm and the Krasnoselskii-Mann iterative algorithm, for solving equilibrium problems, variational inequalities and fixed point problems of multivalued quasi-nonexpansive mapping.

Keywords. Equilibrium problem; Fixed point, Multivalued quasi-nonexpansive mapping; Variational inequality.
\end{abstract}

2010 Mathematics Subject Classification. 47H05, 47H09, 47J25.

\section{INTRODUCTION}

In 1994, Blum and Oettli [3] introduced and studied an equilibrium problem, which has a great impact and influence on the development of several branches of pure and applied sciences, in the setting of infinite dimensional Hilbert spaces. Let $H$ be a Hilbert space and let $K$ be a nonempty, closed and convex subset of $H$. Let $f: K \times K \rightarrow \mathbb{R}$, where $\mathbb{R}$ denotes the set of real numbers, be a bifunction. Recall that the equilibrium problem is to find a point $x^{*} \in K$ such that

$$
f\left(x^{*}, y\right) \geq 0, \quad \forall y \in K .
$$

The solution set of the equilibrium problem is denoted by $E P(f)$ in this paper.

Recently, studies on solutions of the equilibrium problem were extensively carried out in Hilbert spaces and in certain Banach spaces; see, for example, [1, 8, 11, 12, 13, 23, 27] and the references therein. It has been shown that the equilibrium problem provides a novel and unified framework for a wide class of problems which arise in economics, finance, image reconstruction, ecology, transportation, and network. It also has been shown that the equilibrium problem includes variational inequalities, minimax inequalities, the Nash equilibrium, and game theory as special cases. Recently, a lot of iterative algorithms have been studied in infinite dimensional spaces, see $[3,9,25,27,31]$ and the references therein.

Recall that an operator $A: K \rightarrow H$ is said to be monotone iff

$$
\langle A x-A y, x-y\rangle \geq 0, \quad \forall x, y \in K
$$

E-mail address: sowthierno89@ gmail.com.

Received May 18, 2019; Accepted July 9, 2019.

(C)2019 Applied Set-Valued Analysis and Optimization 
$A: K \rightarrow H$ is said to be $\alpha$-inverse strongly monotone iff there exists a constant $\alpha>0$ such that

$$
\langle A x-A y, x-y\rangle \geq \alpha\|A x-A y\|^{2}, \quad \forall x, y \in K .
$$

It is obvious that if $A$ is $\alpha$-inverse strongly monotone, then it is monotone and Lipschitz continuous.

Recall the classical variational inequality problem is to find a point $u \in K$ such that

$$
\langle A u, v-u\rangle \geq 0, \quad \forall v \in K .
$$

We denote the set of solutions of the variational inequality problem by $V I(K, A)$. Variational inequality (1.2) was formulated in the late 1960's by Lions and Stampacchia [15]. Since then, it has been extensively studied via numerical methods. For a lot of real-life problems, such as, in signal processing, resource allocation, image recovery and so on, the constraints can be expressed as the variational inequality problem. Hence, the problem of finding solutions of variational inequality (1.2) has become a flourishing area of contemporary research for numerous mathematicians working in nonlinear operator theory; see, for example, $[10,17,18,28,29,30]$ and the references therein.

It is easy to see that $u \in K$ is a solution of variational inequality (1.2) iff $u$ is a fixed point of the mapping $P_{K}(I-\lambda A)$, where $P_{K}$ is the known metric projection from $H$ onto $K, I$ is the identity mapping and $\lambda$ is some real positive number.

A well known method for solving the variational inequality problem is the projection method which starts with $x_{1} \in K$ and generates a sequence $\left\{x_{n}\right\}$ in the following recursion formula,

$$
x_{n+1}=P_{K}\left(x_{n}-\lambda_{n} A x_{n}\right), \quad n \geq 1,
$$

where $\left\{\lambda_{n}\right\}$ a sequence of positive numbers satisfying appropriate conditions. In the case that $A$ is $\alpha$-inverse strongly monotone, Iiduka and Takahashi [14] proved that the sequence $\left\{x_{n}\right\}$ generated by (1.3) converges weakly to an element of $V I(K, A)$. Spotlights have been shed on the modification of the above projection algorithm so that the norm convergence is guaranteed under mild conditions recent; see $[1,8,10,13,25,27,29]$.

Let $T: K \rightarrow 2^{K}$ be a multivalued mapping. An element $x \in K$ is called a fixed point of $T$ if $x \in T x$. For single valued mapping, this reduces to $T x=x$. The fixed point set of $T$ is denoted by $F(T):=\{x \in$ $D(T): x \in T x\}$. Let $D$ be a nonempty subset of a normed space $E$. The set $D$ is said to be proximinal (see [20]) if for each $x \in E$, there exists $u \in D$ such that

$$
\|x-u\|=\inf \{\|x-y\|: y \in D\}=d(x, D)
$$

Every nonempty, closed and convex subset of a real Hilbert space is proximinal. Let $C B(D), K(D)$ and $P(D)$ denote the family of nonempty closed bounded subsets, nonempty compact subsets, and nonempty proximinal bounded subsets of $D$ respectively. The Pompeiu Hausdorff metric on $C B(D)$ is defined by:

$$
H(A, B)=\max \left\{\sup _{a \in A} d(a, B), \sup _{b \in B} d(b, A)\right\}
$$

for all $A, B \in C B(D)$ (see Berinde [4]). A multi-valued mapping $T: D(T) \subseteq E \rightarrow C B(E)$ is called $L$ Lipschitzian if there exists $L>0$ such that

$$
H(T x, T y) \leq L\|x-y\|, \quad \forall x, y \in D(T)
$$


If $L \in(0,1)$, we say that $T$ is a contraction. $T$ is said to be nonexpansive if $L=1$. A multivalued map $T$ is said to be quasi-nonexpansive if $F(T) \neq \emptyset$ and

$$
H(T x, T p) \leq\|x-p\|, \quad \forall x \in D(T), p \in F(T) .
$$

Remark 1.1. It is easy to see that the class of mulivalued quasi-nonexpansive mappings properly includes that of multivalued nonexpansive maps with fixed points.

Many problems arising in different areas of mathematics such as optimization, variational analysis, differential equations, mathematical economics, and game theory can be modeled as fixed point equations of the form $x \in T x$, where $T$ is a multivalued nonexpansive mapping. There are many effective algorithms for solving the fixed point problem [5, 7, 20, 22]. One of the most efficient methods for approximating fixed points of single-valued nonexpansive mappings dates back to 1953 and is the Mann's method. Recall Mann's method generates a sequence in the following manner

$$
x_{0} \in C, x_{n+1}=\alpha_{n} x_{n}+\left(1-\alpha_{n}\right) T x_{n},
$$

where $\left\{\alpha_{n}\right\}$ is a sequence in $(0,1)$. But Mann's iteration is only weakly convergent.

Recently, Zeng and Yao [32] introduced an extragradient method for finding a common element of the set of fixed points of a nonexpansive mapping and the set of solutions of a variational inequality problem. Indeed, they obtained the following strong convergence theorem.

Theorem 1.1. [32] Let $C$ be a nonempty closed convex subset of a real Hilbert space H. Let $A: C \rightarrow H$ be a monotone $k$-Lipschitz continuous mapping and let $T: C \rightarrow C$ be a nonexpansive mapping such that $F(T) \cap V I(C, A) \neq \emptyset$. Let the sequences $\left\{x_{n}\right\}$ and $\left\{y_{n}\right\}$ be generated by

$$
\left\{\begin{array}{l}
x_{0} \in H, \\
y_{n}=P_{C}\left(x_{n}-\lambda_{n} A x_{n}\right), \\
x_{n+1}=\alpha_{n} x_{0}+\left(1-\alpha_{n}\right) S P_{C}\left(x_{n}-\lambda_{n} A y_{n}\right),
\end{array}\right.
$$

where $\left\{\lambda_{n}\right\}$ and $\left\{\alpha_{n}\right\}$ satisfy the following conditions:

(a) $\left\{\lambda_{n} k\right\} \subset(0,1-\delta)$ for some $\delta \in(0,1)$,

(b) $\left\{\alpha_{n}\right\} \subset(0,1), \sum_{n=0}^{\infty} \alpha_{n}=\infty, \lim _{n \rightarrow \infty} \alpha_{n}=0$.

Then the sequences $\left\{x_{n}\right\}$ and $\left\{y_{n}\right\}$ converge strongly to the same point $P_{F(T) \cap V I(C, A)} x_{0}$ provided that

$$
\lim _{n \rightarrow \infty}\left\|x_{n+1}-x_{n}\right\|=0 .
$$

Remark 1.2. The iterative scheme (1.4) in Theorem 1.1 is strongly convergent with the assumption (1.5) on the sequence $\left\{x_{n}\right\}$.

Recently, Plubtieng and Kumam [21] further studied the common element problem and proved the following result.

Theorem 1.2. Let $K$ be a nonempty closed convex subset of a real Hilbert space $H$ and let $A: K \rightarrow H$ be an $\alpha$-inverse strongly monotone operator. Let $f: K \times K \rightarrow \mathbb{R}$ be a bifunction satisfying (A1)-(A4) such 
that $G:=E P(f) \cap V I(K, A) \neq \emptyset$. Let $\left\{x_{n}\right\}$ be a sequence defined as follows:

$$
\left\{\begin{array}{l}
x_{0} \in K \\
y_{n}=P_{K}\left(I-\theta_{n} A\right) x_{n}, \\
f\left(u_{n}, y\right)+\frac{1}{r_{n}}\left\langle y-u_{n}, u_{n}-y_{n}\right\rangle \geq 0, \forall y \in K \\
x_{n+1}=\alpha_{n} x_{n}+\left(1-\alpha_{n}\right) u_{n},
\end{array}\right.
$$

where $\left\{r_{n}\right\}$ and $\left\{\theta_{n}\right\}$ are positive real sequences and $\left\{\alpha_{n}\right\}$ is real sequences in $(0,1)$. If

(i) $\lim _{n \rightarrow \infty} \alpha_{n}=0, \quad($ ii $) \sum_{n=0}^{\infty}\left|r_{n+1}-r_{n}\right|<\infty$ and $\theta_{n} \in[a, b] \subset(0, \min \{1,2 \alpha\})$,

(iii) $\lim _{n \rightarrow \infty}\left|\theta_{n+1}-\theta_{n}\right|, \lim _{n \rightarrow \infty}\left(1-\alpha_{n}\right) \alpha_{n}=\infty$ and $\lim _{n \rightarrow \infty} \inf r_{n}>0$.

then, the sequences $\left\{x_{n}\right\}$ and $\left\{z_{n}\right\}$ generated by (1.6) converge weakly to $x^{*} \in G$.

Motivated and inspired by the ongoing results in this field, we introduce a new iterative algorithm and prove a strong convergence theorem for equilibrium problem (1.1), variational inequality problem (1.2) and the fixed point problem of a multivalued quasi-nonexpansive mapping in Hilbert spaces.

\section{PRELIMINARIES}

Let $K$ be a nonempty, closed convex subset of $H$. For any $y \in H$, there exists a unique point in $C$, denoted by $P_{K}(u)$, such that

$$
\left\|y-P_{K}(u)\right\| \leq\|y-x\|, \quad \forall x \in K .
$$

It is well known that the projection operator can be characterized by the following two properties

(i) $\left\langle P_{K} y-y, x-P_{K} y\right\rangle \geq 0, \forall x \in K$;

(ii) $\left\langle P_{K} x-P_{K} y, x-y\right\rangle \geq\left\|P_{K} x-P_{K} y\right\|^{2}, \forall x, y \in H$;

(iii) $\left\|P_{K} y-x\right\|^{2} \leq\|y-x\|^{2}-\left\|P_{K} y-y\right\|^{2}, \forall x \in K$.

In the context of variational inequality problem (1.2), we have

$$
u \in V I(K, A) \Longleftrightarrow u=P_{K}(I-\theta A) u, \quad \theta>0 .
$$

For solving equilibrium problem (1.1), let us assume that $f$ satisfies the following conditions:

(A1) $f(x, x)=0$ for all $x \in C$;

(A2) $f$ is monotone, i.e., $f(x, y)+f(y, x) \leq 0$ for all $x, y \in C$;

(A3) for each $x, y, z \in C$,

$$
\lim _{t \rightarrow 0} f(t z+(1-t) x, y) \leq f(x, y)
$$

(A4) for each $x \in C, \quad y \rightarrow f(x, y)$ is convex and lower semicontinuous.

Then, for any $x, y \in H$, the following inequalities hold:

$$
\|x+y\|^{2} \leq\|x\|^{2}+2\langle y, x+y\rangle
$$

and

$$
\|\lambda x+(1-\lambda) y\|^{2}=\lambda\|x\|^{2}+(1-\lambda)\|y\|^{2}-(1-\lambda) \lambda\|x-y\|^{2}, \lambda \in(0,1) .
$$


Lemma 2.1. [7] Let $H$ be a real Hilbert space and let $K$ be a nonempty closed and convex subset of $H$. Let $T: K \rightarrow C B(K)$ be a multivalued nonexpansive mapping with convex-values. Then $I-T$ is demi-closed at zero.

Lemma 2.2. [16] Assume that $\left\{a_{n}\right\}$ is a sequence of nonnegative real numbers such that

$$
a_{n+1} \leq\left(1-\alpha_{n}\right) a_{n}+\alpha_{n} \sigma_{n}
$$

for all $n \geq 0$, where $\left\{\alpha_{n}\right\}$ is a sequence in $(0,1)$ and $\left\{\sigma_{n}\right\}$ is a sequence in $\mathbb{R}$ such that $(a) \sum_{n=0}^{\infty} \alpha_{n}=\infty$, (b) $\lim \sup _{n \rightarrow \infty} \sigma_{n} \leq 0$ or $\sum_{n=0}^{\infty}\left|\sigma_{n} \alpha_{n}\right|<\infty$. Then $\lim _{n \rightarrow \infty} a_{n}=0$.

Lemma 2.3. [19] Let $t_{n}$ be a sequence of real numbers that does not decrease at infinity in a sense that there exists a subsequence $t_{n_{i}}$ of $t_{n}$ such that $t_{n_{i}}$ such that $t_{n_{i}} \leq t_{n_{i+1}}$ for all $i \geq 0$. For sufficiently large numbers $n \in \mathbb{N}$, an integer sequence $\{\tau(n)\}$ is defined as follows:

$$
\tau(n)=\max \left\{k \leq n: t_{k} \leq t_{k+1}\right\} .
$$

Then, $\tau(n) \rightarrow \infty$ as $n \rightarrow \infty$ and

$$
\max \left\{t_{\tau(n)}, t_{n}\right\} \leq t_{\tau(n)+1}
$$

The following lemma appears implicitly in [3].

Lemma 2.4. [3] Let $C$ be a nonempty closed convex subset of $H$ and let $f$ be a bifunction of $C \times C$ into $\mathbb{R}$ satisfying (A1)-(A4). Let $r>0$ and $x \in H$. Then, there exists $z \in C$ such that

$$
f(z, y)+\frac{1}{r}\langle y-z, z-x\rangle \geq 0, \forall y \in C .
$$

The following lemma was given in [6].

Lemma 2.5. [6] Assume that $f: C \times C \rightarrow \mathbb{R}$ is a bifunction satisfying conditions (A1)-(A4). For $r>0$ and $x \in H$, define a mapping $T_{r}: H \rightarrow C$ as follows

$$
T_{r}(x)=\left\{z \in C, f(z, y)+\frac{1}{r}\langle y-z, z-x\rangle \geq 0, \forall y \in C\right\}, \quad \forall x \in H .
$$

Then, the following hold:

1.T $T_{r}$ is single-valued;

2. $T_{r}$ is firmly nonexpansive, i.e., $\left\|T_{r}(x)-T_{r}(y)\right\|^{2} \leq\left\langle T_{r} x-T_{r} y, x-y\right\rangle, \forall x, y \in H$;

3.F $\left(T_{r}\right)=E P(f)$;

4. EP(f) is closed and convex.

Lemma 2.6. [26] Let $K$ be a nonempty closed and convex subset of a real Hilbert space $H$ and let $A$ be a monotone, hemicontinuous map of $K$ into $H$. Let $B \subset H \times H$ be an operator defined as follows:

$$
B z= \begin{cases}A z+N_{K}(z), & \text { if } z \in K, \\ \emptyset, & \text { if } z \notin K,\end{cases}
$$

where $N_{K}(z)$ is the normal cone to $K$ at $z$ and defined by

$$
N_{K}(z)=\{w \in H:\langle w, z-v\rangle \geq 0 \forall v \in K\} .
$$

Then, $B$ is maximal monotone and $B^{-1}(0)=V I(K, A)$. 
Lemma 2.7. [24] Let $H$ be a real Hilbert space and let $K$ be a nonempty, closed convex subset of $H$. Let $A: K \rightarrow H$ be an $\alpha$-inverse strongly monotone mapping. Then, $I-\theta A$ is nonexpansive for all $x, y \in K$ and $\theta \in[0,2 \alpha]$.

\section{Main Results}

Now, we are ready to present our main result.

Theorem 3.1. Let $K$ be a nonempty, closed convex cone of a real Hilbert space $H$ and let $A: K \rightarrow H$ be an $\alpha$-inverse strongly monotone operator. Let $f$ be a bifunction from $K \times K \rightarrow \mathbb{R}$ satisfying (A1)(A4). Let $T: K \rightarrow C B(K)$ be a multivalued quasi-nonexpansive mapping such that $G:=E P(f) \cap F(T) \cap$ $V I(K, A) \neq \emptyset$ and $T p=\{p\}, \forall p \in G$. Let $\left\{x_{n}\right\}$ be a sequence defined as follows:

$$
\left\{\begin{array}{l}
x_{0} \in K \\
f\left(u_{n}, y\right)+\frac{1}{r_{n}}\left\langle y-u_{n}, u_{n}-x_{n}\right\rangle \geq 0, \forall y \in K \\
z_{n}=P_{K}\left(I-\theta_{n} A\right) u_{n} \\
y_{n}=\beta_{n} z_{n}+\left(1-\beta_{n}\right) v_{n}, v_{n} \in T z_{n} \\
x_{n+1}=\alpha_{n}\left(\lambda_{n} x_{n}\right)+\left(1-\alpha_{n}\right) y_{n}
\end{array}\right.
$$

where $\left\{\alpha_{n}\right\},\left\{\beta_{n}\right\},\left\{\lambda_{n}\right\}$ and $\left\{\theta_{n}\right\}$ are real sequences in $(0,1)$, and $\left\{r_{n}\right\}$ is a real positive sequence satisfying the following conditions:

(i) $\lim _{n \rightarrow \infty} \alpha_{n}=0, \quad$ (ii) $\lim _{n \rightarrow \infty} \inf \beta_{n}\left(1-\beta_{n}\right)>0$ and $\theta_{n} \in[a, b] \subset(0, \min \{1,2 \alpha\})$, (iii) $\lim _{n \rightarrow \infty} \lambda_{n}=1, \sum_{n=0}^{\infty}\left(1-\lambda_{n}\right) \alpha_{n}=\infty$ and $\lim _{n \rightarrow \infty} \inf r_{n}>0$.

If $I-T$ is demiclosed at the origin, then, the sequences $\left\{x_{n}\right\}$ and $\left\{z_{n}\right\}$ generated by (3.1) converge strongly to $x^{*}=P_{G}(0)$, where $P_{G}$ is the metric projection from $K$ onto $G$.

Proof. First, we prove that the sequence $\left\{x_{n}\right\}$ is bounded. Let $p \in G$. It follows from Lemma 2.7 that

$$
\left\|z_{n}-p\right\|=\left\|P_{K}\left(I-\theta_{n} A\right) u_{n}-p\right\| \leq\left\|u_{n}-p\right\|=\left\|T_{r_{n}} x_{n}-T_{r_{n}} p\right\| \leq\left\|x_{n}-p\right\|
$$

Since $T$ is quasi-nonexpansive, we have

$$
\begin{aligned}
\left\|y_{n}-p\right\| & \leq \beta_{n}\left\|z_{n}-p\right\|+\left(1-\beta_{n}\right)\left\|v_{n}-p\right\| \\
& \leq \beta_{n}\left\|z_{n}-p\right\|+\left(1-\beta_{n}\right) H\left(T z_{n}, T p\right) \\
& \leq\left\|z_{n}-p\right\| .
\end{aligned}
$$

Hence,

$$
\left\|y_{n}-p\right\| \leq\left\|z_{n}-p\right\| \leq\left\|u_{n}-p\right\| \leq\left\|x_{n}-p\right\|
$$


Using (3.1) and (3.2), we have

$$
\begin{aligned}
\left\|x_{n+1}-p\right\| & =\left\|\alpha_{n}\left(\lambda_{n} x_{n}\right)+\left(1-\alpha_{n}\right) y_{n}-p\right\| \\
& \leq \alpha_{n} \lambda_{n}\left\|x_{n}-p\right\|+\left(1-\alpha_{n}\right)\left\|y_{n}-p\right\|+\left(1-\lambda_{n}\right) \alpha_{n}\|p\| \\
& \leq \alpha_{n} \lambda_{n}\left\|x_{n}-p\right\|+\left(1-\alpha_{n}\right)\left\|x_{n}-p\right\|+\left(1-\lambda_{n}\right) \alpha_{n}\|p\| \\
& \leq\left[1-\left(1-\lambda_{n}\right) \alpha_{n}\right]\left\|x_{n}-p\right\|+\left(1-\lambda_{n}\right) \alpha_{n}\|p\| \\
& \leq \max \left\{\left\|x_{n}-p\right\|,\|p\|\right\} .
\end{aligned}
$$

By induction, it is easy to see that

$$
\left\|x_{n}-p\right\| \leq \max \left\{\left\|x_{0}-p\right\|,\|p\|\right\} .
$$

Hence $\left\{x_{n}\right\}$ is bounded, so are $\left\{z_{n}\right\}$, and $\left\{T x_{n}\right\}$. From (3.1) and (3.2), we have

$$
\begin{aligned}
\left\|y_{n}-p\right\|^{2} & =\left\|\beta_{n} z_{n}+\left(1-\beta_{n}\right) v_{n}-p\right\|^{2} \\
& =\left(1-\beta_{n}\right)\left\|v_{n}-p\right\|^{2}+\beta_{n}\left\|z_{n}-p\right\|^{2}-\beta_{n}\left(1-\beta_{n}\right)\left\|v_{n}-z_{n}\right\|^{2} . \\
& \leq\left(1-\beta_{n}\right) H\left(T z_{n}, T p\right)^{2}+\beta_{n}\left\|z_{n}-p\right\|^{2}-\beta_{n}\left(1-\beta_{n}\right)\left\|z_{n}-v_{n}\right\|^{2}
\end{aligned}
$$

Hence,

$$
\left\|y_{n}-p\right\|^{2} \leq\left\|x_{n}-p\right\|^{2}-\beta_{n}\left(1-\beta_{n}\right)\left\|z_{n}-v_{n}\right\|^{2} .
$$

This implies that

$$
\begin{aligned}
\left\|x_{n+1}-p\right\|^{2} \leq & \left\|\alpha_{n} \lambda_{n}\left(x_{n}-p\right)+\left(1-\alpha_{n}\right)\left(y_{n}-p\right)-\left(1-\lambda_{n}\right) \alpha_{n} p\right\|^{2} \\
\leq & \left\|\alpha_{n}\left(\lambda_{n} x_{n}-\lambda_{n} p\right)+\left(1-\alpha_{n}\right)\left(y_{n}-p\right)\right\|^{2}+2\left(1-\lambda_{n}\right) \alpha_{n}\left\langle p, p-x_{n+1}\right\rangle \\
\leq & \alpha_{n} \lambda_{n}^{2}\left\|x_{n}-p\right\|^{2}+\left(1-\alpha_{n}\right)\left\|y_{n}-p\right\|^{2}+2\left(1-\lambda_{n}\right) \alpha_{n}\left\langle p, p-x_{n+1}\right\rangle \\
\leq & \alpha_{n} \lambda_{n}\left\|x_{n}-p\right\|^{2}+\left(1-\alpha_{n}\right)\left[\left\|x_{n}-p\right\|^{2}-\beta_{n}\left(1-\beta_{n}\right)\left\|z_{n}-v_{n}\right\|^{2}\right] \\
& +2\left(1-\lambda_{n}\right) \alpha_{n}\left\langle p, p-x_{n+1}\right\rangle \\
\leq & {\left[1-\left(1-\lambda_{n}\right) \alpha_{n}\right]\left\|x_{n}-p\right\|^{2}-\left(1-\alpha_{n}\right) \beta_{n}\left(1-\beta_{n}\right)\left\|z_{n}-v_{n}\right\|^{2} } \\
& +2\left(1-\lambda_{n}\right) \alpha_{n}\left\langle p, p-x_{n+1}\right\rangle .
\end{aligned}
$$

Therefore,

$$
\left(1-\alpha_{n}\right) \beta_{n}\left(1-\beta_{n}\right)\left\|z_{n}-v_{n}\right\|^{2} \leq\left\|x_{n}-p\right\|^{2}-\left\|x_{n+1}-p\right\|^{2}+2\left(1-\lambda_{n}\right) \alpha_{n}\left\langle p, p-x_{n+1}\right\rangle .
$$

Since $\left\{x_{n}\right\}$ is bounded, there exists a constant $B>0$ sucht that

$$
\left(1-\lambda_{n}\right)\left\langle p, p-x_{n+1}\right\rangle \leq B, \text { for all } n \geq 0 .
$$

Hence,

$$
\left(1-\alpha_{n}\right) \beta_{n}\left(1-\beta_{n}\right)\left\|z_{n}-v_{n}\right\|^{2} \leq\left\|x_{n}-p\right\|^{2}-\left\|x_{n+1}-p\right\|^{2}+2 \alpha_{n} B .
$$

Now we prove that $\left\{x_{n}\right\}$ converges strongly to $x^{*}$.

We divide the proof into two cases. 
Case 1. Assume that the sequence $\left\{\left\|x_{n}-p\right\|\right\}$ is monotonically decreasing sequence. Then $\left\{\left\|x_{n}-p\right\|\right\}$ is convergent. Clearly, we have

$$
\lim _{n \rightarrow \infty}\left\|x_{n}-p\right\|^{2}-\left\|x_{n+1}-p\right\|^{2}=0
$$

It then implies from (3.5) that

$$
\lim _{n \rightarrow \infty}\left(1-\alpha_{n}\right) \beta_{n}\left(1-\beta_{n}\right)\left\|z_{n}-v_{n}\right\|^{2}=0 .
$$

Using the fact that $\lim _{n \rightarrow \infty} \inf \beta_{n}\left(1-\beta_{n}\right)>0$, we have

$$
\lim _{n \rightarrow \infty}\left\|z_{n}-v_{n}\right\|=0
$$

Hence,

$$
\lim _{n \rightarrow \infty} d\left(z_{n}, T z_{n}\right)=0 .
$$

From (3.1), the convexity of $\|\cdot\|^{2}$ and Lemma 2.7, we have

$$
\begin{aligned}
\left\|x_{n+1}-p\right\|^{2} & =\left\|\alpha_{n}\left(\lambda_{n} x_{n}\right)+\left(1-\alpha_{n}\right) y_{n}-p\right\|^{2} \\
& \leq \alpha_{n}\left\|\left(\lambda_{n} x_{n}\right)-p\right\|^{2}+\left(1-\alpha_{n}\right)\left\|y_{n}-p\right\|^{2} \\
& \leq \alpha_{n}\left\|\left(\lambda_{n} x_{n}\right)-p\right\|^{2}+\left(1-\alpha_{n}\right)\left\|z_{n}-p\right\|^{2} \\
& =\alpha_{n}\left\|\left(\lambda_{n} x_{n}\right)-p\right\|^{2}+\left(1-\alpha_{n}\right)\left\|P_{K}\left(I-\theta_{n} A\right) u_{n}-P_{K}\left(I-\theta_{n} A\right) p\right\|^{2} \\
& \leq \alpha_{n}\left\|\left(\lambda_{n} x_{n}\right)-p\right\|^{2}+\left(1-\alpha_{n}\right)\left[\left\|u_{n}-p\right\|^{2}+\theta_{n}\left(\theta_{n}-2 \alpha\right)\left\|A u_{n}-A p\right\|^{2}\right] \\
& \leq \alpha_{n}\left\|\left(\lambda_{n} x_{n}\right)-p\right\|^{2}+\left(1-\alpha_{n}\right)\left\|x_{n}-p\right\|^{2}+\left(1-\alpha_{n}\right) a(b-2 \alpha)\left\|A u_{n}-A p\right\|^{2} .
\end{aligned}
$$

Therefore,

$$
\left(1-\alpha_{n}\right) a(2 \alpha-b)\left\|A u_{n}-A p\right\|^{2} \leq\left\|x_{n}-p\right\|^{2}-\left\|x_{n+1}-p\right\|^{2}+\alpha_{n}\left\|\left(\lambda_{n} x_{n}\right)-p\right\|^{2} .
$$

Since $\alpha_{n} \rightarrow 0$ as $n \rightarrow \infty$, and $\left\{x_{n}\right\}$ is bounded, we obtain

$$
\lim _{n \rightarrow \infty}\left\|A u_{n}-A p\right\|^{2}=0
$$

On the other hand, we have

$$
\begin{aligned}
& \left\|z_{n}-p\right\|^{2} \\
= & \left\|P_{K}\left(I-\theta_{n} A\right) u_{n}-P_{K}\left(I-\theta_{n} A\right) p\right\|^{2} \\
\leq & \left\langle z_{n}-p,\left(I-\theta_{n} A\right) u_{n}-\left(I-\theta_{n} A\right) p\right\rangle \\
= & \frac{1}{2}\left[\left\|\left(I-\theta_{n} A\right) u_{n}-\left(I-\theta_{n} A\right) p\right\|^{2}+\left\|z_{n}-p\right\|^{2}-\left\|\left(I-\theta_{n} A\right) u_{n}-\left(I-\theta_{n} A\right) p-\left(z_{n}-p\right)\right\|^{2}\right] \\
\leq & \frac{1}{2}\left[\left\|u_{n}-p\right\|^{2}+\left\|z_{n}-p\right\|^{2}-\left\|u_{n}-z_{n}\right\|^{2}+2 \theta_{n}\left\langle z_{n}-p, A u_{n}-A p\right\rangle-\theta_{n}{ }^{2}\left\|A u_{n}-A p\right\|^{2}\right] \\
\leq & \frac{1}{2}\left[\left\|x_{n}-p\right\|^{2}+\left\|z_{n}-p\right\|^{2}-\left\|u_{n}-z_{n}\right\|^{2}+2 \theta_{n}\left\langle z_{n}-p, A u_{n}-A p\right\rangle-\theta_{n}{ }^{2}\left\|A u_{n}-A p\right\|^{2}\right] .
\end{aligned}
$$

So,

$$
\left\|z_{n}-p\right\|^{2} \leq\left\|x_{n}-p\right\|^{2}-\left\|u_{n}-z_{n}\right\|^{2}+2 \theta_{n}\left\langle z_{n}-p, A u_{n}-A p\right\rangle-\theta_{n}{ }^{2}\left\|A u_{n}-A p\right\|^{2},
$$


which implies

$$
\begin{aligned}
\left\|x_{n+1}-p\right\|^{2} & \leq \alpha_{n}\left\|\left(\lambda_{n} x_{n}\right)-p\right\|^{2}+\left(1-\alpha_{n}\right)\left\|y_{n}-p\right\|^{2} \\
& \leq \alpha_{n}\left\|\left(\lambda_{n} x_{n}\right)-p\right\|^{2}+\left(1-\alpha_{n}\right)\left\|z_{n}-p\right\|^{2} \\
& \leq \alpha_{n}\left\|\left(\lambda_{n} x_{n}\right)-p\right\|^{2}+\left\|x_{n}-p\right\|^{2}-\left(1-\alpha_{n}\right)\left\|u_{n}-z_{n}\right\|^{2}-\left(1-\alpha_{n}\right) \theta_{n}{ }^{2}\left\|A u_{n}-A p\right\|^{2} \\
& +2 \theta_{n}\left(1-\alpha_{n}\right)\left\langle z_{n}-p, A u_{n}-A p\right\rangle .
\end{aligned}
$$

Since $\alpha_{n} \rightarrow 0$ as $n \rightarrow \infty$, and (3.10), we obtain

$$
\lim _{n \rightarrow \infty}\left\|u_{n}-z_{n}\right\|^{2}=0 .
$$

Letting $p \in G$, we have

$$
\begin{aligned}
\left\|u_{n}-p\right\|^{2} & =\left\|T_{r_{n}} x_{n}-T_{r_{n}} p\right\|^{2} \\
& \leq\left\langle T_{r_{n}} x_{n}-T_{r_{n}} p, x_{n}-p\right\rangle \\
& \leq\left\langle u_{n}-p, x_{n}-p\right\rangle \\
& =\frac{1}{2}\left(\left\|u_{n}-p\right\|^{2}+\left\|x_{n}-p\right\|^{2}-\left\|x_{n}-u_{n}\right\|^{2}\right)
\end{aligned}
$$

and hence

$$
\left\|u_{n}-p\right\|^{2} \leq\left\|x_{n}-p\right\|^{2}-\left\|x_{n}-u_{n}\right\|^{2} .
$$

Therefore, from (3.1), and (3.12), we get that

$$
\begin{aligned}
\left\|x_{n+1}-p\right\|^{2} \leq & \left\|\alpha_{n}\left(\left(\lambda_{n} x_{n}\right)-p\right)+\left(1-\alpha_{n}\right)\left(y_{n}-p\right)\right\|^{2} \\
\leq & \left(1-\alpha_{n}\right)^{2}\left\|y_{n}-p\right\|^{2}+2 \alpha_{n}\left\langle\left(\lambda_{n} x_{n}\right)-p, x_{n+1}-p\right\rangle \\
\leq & \left(1-\alpha_{n}\right)^{2}\left\|u_{n}-p\right\|^{2}+2 \alpha_{n} \lambda_{n}\left\langle x_{n}-p, x_{n+1}-p\right\rangle+2\left(1-\lambda_{n}\right) \alpha_{n}\left\langle p, x_{n+1}-p\right\rangle \\
\leq & \left(1-2 \alpha_{n}+\alpha_{n}^{2}\right)\left\|x_{n}-p\right\|^{2}-\left(1-\alpha_{n}\right)^{2}\left\|x_{n}-u_{n}\right\|^{2}+2 \alpha_{n} \lambda_{n}\left\|x_{n}-p\right\|\left\|x_{n+1}-p\right\| \\
& +2 \alpha_{n}\left(1-\lambda_{n}\right)\|p\|\left\|x_{n+1}-p\right\| \\
\leq & \left\|x_{n}-p\right\|^{2}+\alpha_{n}\left\|x_{n}-p\right\|^{2}-\left(1-\alpha_{n}\right)^{2}\left\|x_{n}-u_{n}\right\|^{2}+2 \alpha_{n} \lambda_{n}\left\|x_{n}-p\right\|\left\|x_{n+1}-p\right\| \\
& +2 \alpha_{n}\left(1-\lambda_{n}\right)\|p\|\left\|x_{n+1}-p\right\| .
\end{aligned}
$$

In view of (3.6), we have

$$
\lim _{n \rightarrow \infty}\left\|x_{n}-u_{n}\right\|=0
$$

Using (3.11) and (3.13), we have

$$
\lim _{n \rightarrow \infty}\left\|x_{n}-z_{n}\right\|=0
$$

Since $H$ is reflexive and $\left\{x_{n}\right\}$ is bounded, there exists a subsequence $\left\{x_{n_{k}}\right\}$ of $\left\{x_{n}\right\}$ such that $\left\{x_{n_{k}}\right\}$ converges weakly to $\omega$ in $K$ and

$$
\limsup _{n \rightarrow+\infty}\left\langle x^{*}, x^{*}-x_{n}\right\rangle=\lim _{k \rightarrow+\infty}\left\langle x^{*}, x^{*}-x_{n_{k}}\right\rangle .
$$

From (3.9) and the fact that $I-T$ is demiclosed, we obtain $\omega \in F(T)$. Let us show $\omega \in \operatorname{VI}(K, A)$.

Now, let us introduce a multivalued map $B: H \rightarrow 2^{H}$ defined by

$$
B z= \begin{cases}A z+N_{K}(z), & z \in K, \\ \emptyset, & z \notin K,\end{cases}
$$


where $N_{K}(z)$ is the normal $K$ at $z$ defined by

$$
N_{K}(z)=\{w \in H:\langle w, z-v\rangle \geq 0, \quad \forall, v \in K\} .
$$

From Lemma 2.6, we have that $B$ is maximal monotone and $B^{-1}(0)=V I(K, A)$. Let $(u, v) \in G(A)$. Since $v-A u \in N_{K}(u)$ and $z_{n} \in K$, we have

$$
\left\langle u-z_{n}, v-A u\right\rangle \geq 0
$$

On other hand, from $z_{n}=P_{K}\left(I-\theta_{n} A\right) u_{n}$, we have, $\left\langle u-z_{n}, z_{n}-\left(I-\theta_{n} A\right) u_{n}\right\rangle \geq 0$. Hence

$$
\left\langle u-z_{n}, \frac{z_{n}-u_{n}}{\theta_{n}}+A u_{n}\right\rangle \geq 0 .
$$

Therefore,

$$
\begin{aligned}
\left\langle u-z_{n_{k}}, v\right\rangle & \geq\left\langle u-z_{n_{k}}, A u\right\rangle \\
& \geq\left\langle u-z_{n_{k}}, A u\right\rangle-\left\langle u-z_{n_{k}}, \frac{z_{n_{k}}-u_{n_{k}}}{\theta_{n_{k}}}+A u_{n_{k}}\right\rangle \\
& \geq\left\langle u-z_{n_{k}}, A u-A z_{n_{k}}\right\rangle+\left\langle u-z_{n_{k}}, A z_{n_{k}}-A u_{n_{k}}\right\rangle-\left\langle u-z_{n_{k}}, \frac{z_{n_{k}}-u_{n_{k}}}{\theta_{n_{k}}}\right\rangle \\
& \geq\left\langle u-z_{n_{k}}, A z_{n_{k}}-A u_{n_{k}}\right\rangle-\left\langle u-z_{n_{k}}, \frac{z_{n_{k}}-u_{n_{k}}}{\theta_{n_{k}}}\right\rangle .
\end{aligned}
$$

By using the fact that $A$ is $\frac{1}{\alpha}$-Lipschitz, we have

$$
\left\langle u-z_{n_{k}}, v\right\rangle \geq-N\left(\frac{\left\|z_{n_{k}}-u_{n_{k}}\right\|}{\alpha}+\frac{\left\|z_{n_{k}}-u_{n_{k}}\right\|}{a}\right) .
$$

where $N$ is a positive constant such that $\sup _{k \geq 1}\left\{\left\|u-z_{n_{k}}\right\|\right\} \leq M$. Since $z_{n_{k}} \rightarrow \omega$, it follows from (3.11) that $\langle u-\omega, v\rangle \geq 0$ as $k \rightarrow \infty$. Since $B$ is maximal monotone, we have $\omega \in B^{-1}(0)$ and we obtain that $\omega \in \operatorname{VI}(K, A)$. By using (3.13), we have $u_{n_{k}} \rightarrow \omega$. Let us show $\omega \in E P(f)$. It follows by Lemma 2.4 and (A2) that $\frac{1}{r_{n}}\left\langle y-u_{n}, u_{n}-x_{n}\right\rangle \geq f\left(y, u_{n}\right)$. Hence

$$
\left\langle y-u_{n_{k}}, \frac{u_{n_{k}}-x_{n_{k}}}{r_{n_{k}}}\right\rangle \geq f\left(y, u_{n_{k}}\right)
$$

Since $\frac{u_{n_{k}}-x_{n_{k}}}{r_{n_{k}}} \rightarrow 0$ and $u_{n_{k}} \rightarrow \omega$, it follows $(A 4)$ that $f(y, \omega) \leq 0$ for all $y \in K$. For $t$ with $0<t<1$ and $y \in K$, since $y \in K$ and $\omega \in K$, we have $y_{t} \in K$, where $y_{t}=t y+(1-t) \omega$. Hence $f\left(y_{t}, \omega\right) \leq 0$. So, from $(A 1)$ and $(A 4)$, we have

$$
0=f\left(y_{t}, y_{t}\right) \leq t f\left(y_{t}, y\right)+(1-t) f\left(y_{t}, \omega\right) \leq t f\left(y_{t}, y\right) .
$$

It follows that $0 \leq f\left(y_{t}, y\right)$. From $(A 3)$, we have $f(\omega, y) \geq 0$ for all $y \in K$ and hence $\omega \in E P(f)$. Therefore, $\omega \in G$. From $x^{*}=P_{G}(0)$, we have

$$
\begin{aligned}
\limsup _{n \rightarrow+\infty}\left\langle x^{*}, x^{*}-x_{n}\right\rangle & =\lim _{k \rightarrow+\infty}\left\langle x^{*}, x^{*}-x_{n_{k}}\right\rangle \\
& \left.=\left\langle x^{*}, x^{*}-\omega\right)\right\rangle \leq 0 .
\end{aligned}
$$


Finally, we show that $x_{n} \rightarrow x^{*}$. From (3.1), we get that

$$
\begin{aligned}
& \left\|x_{n+1}-x^{*}\right\|^{2} \\
= & \left\langle x_{n+1}-x^{*}, x_{n+1}-x^{*}\right\rangle=\alpha_{n} \lambda_{n}\left\langle x_{n}-x^{*}, x_{n+1}-x^{*}\right\rangle+\left(1-\lambda_{n}\right) \alpha_{n}\left\langle x^{*}, x^{*}-x_{n+1}\right\rangle \\
+ & \left(1-\alpha_{n}\right)\left\langle y_{n}-x^{*}, x_{n+1}-x^{*}\right\rangle \\
\leq & \alpha_{n} \lambda_{n}\left\langle x_{n}-x^{*}, x_{n+1}-x^{*}\right\rangle+\left(1-\lambda_{n}\right) \alpha_{n}\left\langle x^{*}, x^{*}-x_{n+1}\right\rangle+\left(1-\alpha_{n}\right)\left\|y_{n}-x^{*}\right\|\left\|x_{n+1}-x^{*}\right\| \\
\leq & {\left[1-\left(1-\lambda_{n}\right) \alpha_{n}\right]\left\|x_{n}-x^{*}\right\|\left\|x_{n+1}-x^{*}\right\|+\left(1-\lambda_{n}\right) \alpha_{n}\left\langle x^{*}, x^{*}-x_{n+1}\right\rangle } \\
\leq & \frac{1-\left(1-\lambda_{n}\right) \alpha_{n}}{2}\left(\left\|x_{n}-x^{*}\right\|^{2}+\left\|x_{n+1}-x^{*}\right\|^{2}\right)+\left(1-\lambda_{n}\right) \alpha_{n}\left\langle x^{*}, x^{*}-x_{n+1}\right\rangle,
\end{aligned}
$$

which implies that

$$
\left\|x_{n+1}-x^{*}\right\|^{2} \leq\left[1-\left(1-\lambda_{n}\right) \alpha_{n}\right]\left\|x_{n}-x^{*}\right\|+2\left(1-\lambda_{n}\right) \alpha_{n}\left\langle x^{*}, x^{*}-x_{n+1}\right\rangle .
$$

We can check that all the assumptions of Lemma 2.2 are satisfied. Therefore, we deduce $x_{n} \rightarrow x^{*}$.

Case 2. Assume that the sequence $\left\{\left\|x_{n}-x^{*}\right\|\right\}$ is not monotonically decreasing sequence.

Let $B_{n}=\left\|x_{n}-x^{*}\right\|$ and $\tau: \mathbb{N} \rightarrow \mathbb{N}$ be a mapping for all $n \geq n_{0}$ (for some $n_{0}$ large enough) by $\tau(n)=$ $\max \left\{k \in \mathbb{N}: k \leq n, B_{k} \leq B_{k+1}\right\}$. We have $\tau$ is a non-decreasing sequence such that $\tau(n) \rightarrow \infty$ as $n \rightarrow \infty$ and $B_{\tau(n)} \leq B_{\tau(n)+1}$ for $n \geq n_{0}$. From (3.5), we have

$$
\left(1-\alpha_{\tau(n)}\right) \beta_{\tau(n)}\left(1-\beta_{\tau(n)}\right)\left\|z_{\tau(n)}-v_{\tau(n)}\right\|^{2} \leq 2 \alpha_{\tau(n)} B \rightarrow 0 \text { as } n \rightarrow \infty .
$$

Furthermore, we have $\left\|z_{\tau(n)}-v_{\tau(n)}\right\| \rightarrow 0$ as $n \rightarrow \infty$. Hence,

$$
\lim _{n \rightarrow \infty} d\left(z_{\tau(n)}, T z_{\tau(n)}\right)=0
$$

By the same argument as in case 1 , we can show that $x_{\tau(n)}$ converges weakly in $H$ and

$$
\limsup _{n \rightarrow+\infty}\left\langle x^{*}, x^{*}-x_{\tau(n)}\right\rangle \leq 0 \text {. }
$$

We have, for all $n \geq n_{0}$,

$$
0 \leq\left\|x_{\tau(n)+1}-x^{*}\right\|^{2}-\left\|x_{\tau(n)}-x^{*}\right\|^{2} \leq\left(1-\lambda_{\tau(n)}\right) \alpha_{\tau(n)}\left[-\left\|x_{\tau(n)}-x^{*}\right\|^{2}+2\left\langle x^{*}, x^{*}-x_{\tau(n)+1}\right\rangle\right],
$$

which implies that

$$
\left\|x_{\tau(n)}-x^{*}\right\|^{2} \leq 2\left\langle x^{*}, x^{*}-x_{\tau(n)+1}\right\rangle .
$$

Then, $\lim _{n \rightarrow \infty}\left\|x_{\tau(n)}-x^{*}\right\|^{2}=0$ and $\lim _{n \rightarrow \infty} B_{\tau(n)}=\lim _{n \rightarrow \infty} B_{\tau(n)+1}=0$. Using Lemma 2.3, we conclude that

$$
0 \leq B_{n} \leq \max \left\{B_{\tau(n)}, B_{\tau(n)+1}\right\}=B_{\tau(n)+1} .
$$

Hence, $\lim _{n \rightarrow \infty} B_{n}=0$, that is, $\left\{x_{n}\right\}$ converges strongly to $x^{*}$. This completes the proof.

If $T$ is not multivalued quasi-nonexpansive, then the condition that $I-T$ is demiclosed can be removed. 
Theorem 3.2. Let $K$ be a nonempty, closed convex cone of a real Hilbert space $H$ and let $A: K \rightarrow H$ be an $\alpha$-inverse strongly monotone operator. Let $f$ be a bifunction from $K \times K \rightarrow \mathbb{R}$ satisfying (A1)(A4). Let $T: K \rightarrow C B(K)$ be a multivalued nonexpansive mapping with convex-values such that $G:=$ $E P(f) \cap F(T) \cap V I(K, A) \neq \emptyset$ and $T p=\{p\}, \forall p \in G$. Let $\left\{x_{n}\right\}$ be a sequence defined as follows:

$$
\left\{\begin{array}{l}
x_{0} \in K, \\
f\left(u_{n}, y\right)+\frac{1}{r_{n}}\left\langle y-u_{n}, u_{n}-x_{n}\right\rangle \geq 0, \forall y \in K \\
z_{n}=P_{K}\left(I-\theta_{n} A\right) u_{n}, \\
y_{n}=\beta_{n} z_{n}+\left(1-\beta_{n}\right) v_{n}, v_{n} \in T z_{n}, \\
x_{n+1}=\alpha_{n}\left(\lambda_{n} x_{n}\right)+\left(1-\alpha_{n}\right) y_{n},
\end{array}\right.
$$

where $\left\{\alpha_{n}\right\},\left\{\beta_{n}\right\},\left\{\lambda_{n}\right\}$ and $\left\{\theta_{n}\right\}$ are real sequences in $(0,1)$, and $\left\{r_{n}\right\}$ is a real positive sequence satisfying the following conditions:

(i) $\lim _{n \rightarrow \infty} \alpha_{n}=0, \quad$ (ii) $\lim _{n \rightarrow \infty} \inf \beta_{n}\left(1-\beta_{n}\right)>0$ and $\theta_{n} \in[a, b] \subset(0, \min \{1,2 \alpha\})$,

(iii) $\lim _{n \rightarrow \infty} \lambda_{n}=1, \sum_{n=0}^{\infty}\left(1-\lambda_{n}\right) \alpha_{n}=\infty$ and $\lim _{n \rightarrow \infty} \inf r_{n}>0$.

Then, the sequences $\left\{x_{n}\right\}$ and $\left\{z_{n}\right\}$ generated by (3.17) converge strongly to $x^{*}=P_{G}(0)$, where $P_{G}$ is the metric projection from $K$ onto $G$.

Proof. Since every multivalued nonexpansive mapping with fixed points is multivalued quasi-nonexpansive, we can obtain from Lemma 2.1 and Theorem 3.1 the desired conclusion easily.

Remark 3.1. In our theorems, we assume that $K$ is a cone. In some cases, for example, if $K$ is the closed unit ball, we can weaken this assumption to the following: $\lambda x \in K$ for all $\lambda \in(0,1)$ and $x \in K$. Therefore, our results can be used to approximate a common element of the set of fixed points of multivalued quasinonexpansive mapping, the set of solutions of variational inequality problems and the set of solutions of equilibrium problems from the closed unit ball to itself.

Corollary 3.1. Let $H$ be a real Hilbert space and let $B$ be a closed unit ball of $H$. Let $A: B \rightarrow H$ be an $\alpha$ inverse strongly monotone operator and let $f$ be a bifunction from $B \times B \rightarrow \mathbb{R}$ satisfying $(A 1)-(A 4)$. Let $T: B \rightarrow C B(B)$ be a multivalued quasi-nonexpansive mapping such that $G:=E P(f) \cap F(T) \cap V I(K, A) \neq$ $\emptyset$ and $T p=\{p\}, \forall p \in G$. Let $\left\{x_{n}\right\}$ be a sequence defined as follows:

$$
\left\{\begin{array}{l}
x_{0} \in B \\
f\left(u_{n}, y\right)+\frac{1}{r_{n}}\left\langle y-u_{n}, u_{n}-x_{n}\right\rangle \geq 0, \forall y \in B \\
z_{n}=P_{K}\left(I-\theta_{n} A\right) u_{n}, \\
y_{n}=\beta_{n} z_{n}+\left(1-\beta_{n}\right) v_{n}, v_{n} \in T z_{n}, \\
x_{n+1}=\alpha_{n}\left(\lambda_{n} x_{n}\right)+\left(1-\alpha_{n}\right) y_{n}
\end{array}\right.
$$


where $\left\{\alpha_{n}\right\},\left\{\beta_{n}\right\},\left\{\lambda_{n}\right\}$ and $\left\{\theta_{n}\right\}$ are real sequences in $(0,1)$, and $\left\{r_{n}\right\}$ is a real positive sequence satisfying the following conditions:

(i) $\lim _{n \rightarrow \infty} \alpha_{n}=0, \quad$ (ii) $\lim _{n \rightarrow \infty} \inf \beta_{n}\left(1-\beta_{n}\right)>0$ and $\theta_{n} \in[a, b] \subset(0, \min \{1,2 \alpha\})$,

(iii) $\lim _{n \rightarrow \infty} \lambda_{n}=1, \sum_{n=0}^{\infty}\left(1-\lambda_{n}\right) \alpha_{n}=\infty$ and $\lim _{n \rightarrow \infty} \inf r_{n}>0$.

If $I-T$ is demiclosed at the origin, then the sequences $\left\{x_{n}\right\}$ and $\left\{z_{n}\right\}$ generated by (3.18) converge strongly to $x^{*}=P_{G}(0)$, where $P_{G}$ is the metric projection from $K$ onto $G$.

Finally, we consider the following optimization problem:

$$
\min _{x \in K} g(x),
$$

where $K$ is a nonempty, closed convex cone of a real Hilbert space $H$ and $g$ is a continuously Fréchet differentiable, convex functional on $K$. We denote the set of solutions of Problem (3.19) by $\Omega$.

Lemma 3.1. [2] Let $H$ be a real Hilbert space. Let $g$ be a continuously Fréchet differentiable, convex functional on $H$ and $\nabla g$ the gradient of $g$. If $\nabla g$ is $\frac{1}{\alpha}$-Lipschitz continuous, then $\nabla g$ is $\alpha$-inverse strongly monotone.

Theorem 3.3. Let $K$ be a nonempty, closed convex cone of a real Hilbert space $H$. Let $g: K \rightarrow \mathbb{R}$ be a continuously Fréchet differentiable, convex functional on $K$ with a $\frac{1}{\alpha}$-Lipschitz continuous $\nabla g$. Let $f$ be a bifunction from $K \times K \rightarrow \mathbb{R}$ satisfying (A1)-(A4). Let $T: K \rightarrow C B(K)$ be a multivalued quasinonexpansive mapping such that $E P(f) \cap F(T) \cap \Omega \neq \emptyset$ and $T p=\{p\}, \forall p \in E P(f) \cap F(T) \cap \Omega$. Let $\left\{x_{n}\right\}$ be a sequence defined as follows:

$$
\left\{\begin{array}{l}
x_{0} \in K \\
f\left(u_{n}, y\right)+\frac{1}{r_{n}}\left\langle y-u_{n}, u_{n}-x_{n}\right\rangle \geq 0, \forall y \in K \\
z_{n}=P_{K}\left(I-\theta_{n} \nabla g\right) u_{n} \\
y_{n}=\beta_{n} z_{n}+\left(1-\beta_{n}\right) v_{n}, v_{n} \in T z_{n} \\
x_{n+1}=\alpha_{n}\left(\lambda_{n} x_{n}\right)+\left(1-\alpha_{n}\right) y_{n}
\end{array}\right.
$$

where $\left\{\beta_{n}\right\},\left\{\lambda_{n}\right\},\left\{\theta_{n}\right\}$ and $\left\{\alpha_{n}\right\}$ are sequences in $(0,1)$ satisfying the following conditions:

(i) $\lim _{n \rightarrow \infty} \alpha_{n}=0, \quad($ ii $) \lim _{n \rightarrow \infty} \inf \beta_{n}\left(1-\beta_{n}\right)>0$ and $\theta_{n} \in[a, b] \subset(0, \min \{1,2 \alpha\})$,

(iii) $\lim _{n \rightarrow \infty} \lambda_{n}=1$ and $\sum_{n=0}^{\infty}\left(1-\lambda_{n}\right) \alpha_{n}=\infty$.

If $I-T$ is demiclosed at the origin, then, the sequences $\left\{x_{n}\right\}$ and $\left\{z_{n}\right\}$ generated by (3.20) converge strongly to an element of $E P(f) \cap F(T) \cap \Omega$.

Proof. Using the properties of $f$, it follows from Lemma 3.1 that $\nabla f$ is $\alpha$-inverse strongly monotone on $K$. It is known that a necessary condition of optimality for a point $x^{*} \in K$ to be a solution of the minimization problem (3.19) is that $x^{*}$ solves variational inequality problem $V I(K, \nabla g)$. This completes the proof. 


\section{REFERENCES}

[1] P.N. Anh, L.T. H. An, The subgradient extragradient method extended to equilibrium problems, Optimization, 64 (2015), 225-248.

[2] J. B. Baillon, G. Haddad, Quelques propriétés des opérateurs angle-bornés et n-cycliquement monotones, Israel J. Math. 26 (1977), 137-150.

[3] E. Blum, W. Oettli, From optimization and variational inequalities to equilibrium problems, Math. Stud. 63 (1994), 123145.

[4] V. Berinde, M. Pcurar, The role of the Pompeiu-Hausdorff metric in fixed point theory, Creative Math. Inform. 22 (2013), 143-150.

[5] A. Bunyawat, S. Suantai, Hybrid methods for a mixed equilibrium problem and fixed points of a countable family of multivalued nonexpansive mappings, Fixed Point Theory Appl. 2013 (2013), Article ID 236.

[6] P.L. Combettes, S.A. Hirstoaga, Equilibrium programming in Hilbert spaces, J. Nonlinear Convex Anal. 6 (2005), 117136.

[7] S.S. Chang, et al., Convergence theorems for some multivalued generalized nonexpansive mappings, Fixed Point Theory Appl. 2014 (2014), Article ID 33.

[8] S.S. Chang, H.W.J. Lee, C.K. Chan, A new method for solving equilibrium problem fixed point problem and variational inequality problem with application to optimization, Nonlinear Anal. 70 (2009), 3307-3319.

[9] S.Y. Cho, X. Qin, On the strong convergence of an iterative process for asymptotically strict pseudocontractions and equilibrium problems, Appl. Math. Comput. 235 (2014), 430-438.

[10] S.Y. Cho, S.M. Kang, Approximation of common solutions of variational inequalities via strict pseudocontractions, Acta Math. Sci. 32 (2012), 1607-1618.

[11] S.Y. Cho, Strong convergence analysis of a hybrid algorithm for nonlinear operators in a Banach space, J. Appl. Anal. Comput. 8 (2018), 19-31.

[12] C.E. Chidume, M.O. Nnakwe, Iterative algorithms for split variational Inequalities and generalized split feasibility problems with applications, J. Nonlinear Var. Anal. 3 (2019), 127-140.

[13] H. He, S. Liu, H. Zhou, An explicit method for finding common solutions of variational inequalities and systems of equilibrium problems and fixed points of an infinite family of nonexpansive mappings, Nonlinear Anal. 72 (2010), 31243135 .

[14] H. Iiduka, W. Takahashi, M. Toyoda, Approximation of solutions of variational inequalities for monotone mappings, PanAmer. Math. J. 14 (2004), 49-61.

[15] J.L. Lions, G. Stampacchia, Variational inequalities, Comm. Pure Appl. Math. 20 (1967), 493-519.

[16] L.S. Liu, Ishikawa and Mann iterative processes with errors for nonlinear strongly accretive mapping in Banach spaces, J. Math. Anal. Appl. 194 (1995), 114-125.

[17] P.E. Maingé, Projected subgradient techniques and viscosity methods for optimization with variational inequality constraints, Eur. J. Oper. Res. 205 (2010), 501-506.

[18] P.E. Maingé, A hybrid extragradient-viscosity method for monotone operators and fixed point problems, SIAM J. Control Optim. 47 (2008), 1499-1515.

[19] P. E. Maingé, Strong convergence of projected subgradient methods for nonsmooth and nonstrictly convex minimization, Set-Valued Anal. 16 (2008), 899-912.

[20] B. Panyanak, Mann and Ishikawa iteration processes for multi-valued mappings in Banach Spaces, Comput. Math. Appl. 54 (2007), 872-877.

[21] S. Plubtieng, P. Kumam, Weak convergence theorems for monotone mappings and countable family of nonexpansive mappings, Comput. Appl. Math. 224 (2009), 614-621.

[22] W. Phuengrattana, K. Lerkchaiyaphum, On solving the split generalized equilibrium problem and the fixed point problem for a countable family of nonexpansive multivalued mappings, Fixed Point Theory Appl. 2018 (2018), Article ID 6.

[23] X. Qin, S.Y. Cho, S.M. Kang, Convergence theorems of common elements for equilibrium problems and fixed point problems in Banach spaces, J. Comput. Appl. Math. 225 (2009), 20-30.

[24] X. Qin, S.S. Chang, Y.J. Cho, Iterative methods for generalized equilibrium problems and fixed point problems with applications, Nonlinear Anal. 11 (2010), 2963-2972. 
[25] X. Qin, M. Shang, Y. Su, A general iterative method for equilibrium problems and fixed point problems in Hilbert spaces, Nonlinear Anal. 69 (2008), 3897-3909.

[26] R.T. Rockafellar, On the maximality of sums of nonlinear monotone operators. Trans. Amer. Math. Soc. 149 (1970), 75-88.

[27] N. Shahzad, H. Zegeye, Convergence theorems of common solutions for fixed point, variational inequality and equilibrium problems, J. Nonlinear Var. Anal. 3 (2019), 189-203.

[28] T.M.M. Sow, A new general iterative algorithm for solving a variational inequality problem with a quasi-nonexpansive mapping in Banach spaces, Commun. Optim. Theory 2019 (2019), Article ID 9.

[29] M. Tian, B.N. Jiang, Weak convergence theorem for zero points of inverse strongly monotone mapping and fixed points of nonexpansive mapping in Hilbert space, Optimization, 66 (2017), 1689-1698.

[30] M. Tian, M. Tong, A self-adaptive Armijo-like step size method for solving monotone variational inequality problems in Hilbert spaces, J. Nonlinear Funct. Anal. 2019 (2019), Article ID 29.

[31] S. Wang, Y. Zhang, W. Wang, H. Guo, Extragradient algorithms for split pseudomonotone equilibrium problems and fixed point problems in Hilbert spaces, J. Nonlinear Funct. Anal. 2019 (2019), Article ID 26.

[32] L.-C. Zeng, J.-C. Yao, Strong convergence theorem by an extragradient method for fixed point problems and variational inequality problems, Taiwanese J. Math. 10 (2006), 1293-1303. 